\title{
Authors' Response: COVID-19 in Children with Asthma
}

\author{
Grigorios Chatziparasidis $^{1}$ (1) $\cdot$ Ahmad Kantar $^{2}$
}

Received: 11 July 2021 / Accepted: 12 July 2021 / Published online: 11 August 2021

(c) The Author(s), under exclusive licence to Springer Science+Business Media, LLC, part of Springer Nature 2021

We appreciate Dr. Öner Özdemir's thoughtful letter in response to our article "COVID-19 in Children with Asthma" [1]. We absolutely agree it is necessary to conduct large-scale epidemiologic studies, clinical trials, and mechanistic studies to disentangle the risk or protective factors for modulating the susceptibility of children with asthma to SARS-CoV-2 infection.

With regard to misconceptions, the author writes that our claims about the potential mechanisms of the mild course of COVID-19 in children obfuscate the multisystem inflammatory syndrome (MIS-C). Recent studies reveal that the majority of children with COVID-19 either remain asymptomatic or present with mild symptomatology [2]. Data from large epidemiological studies showed that only $1 \%-2 \%$ of the recorded SARS-CoV-2 cases [3-5] and less than $1 \%$ of hospital admissions [3-7] involved children. A systemic review from 26 countries reported an incidence of MIS-C of about $0.14 \%$ among all children with COVID19. Since these COVID-19 cases are underestimated, the incidence of MIS-C may be imprecise [8]. Some clinical features of pediatric MIS-C overlap with those of Kawasaki disease and toxic shock syndrome, but some characteristics are unique. Though rare, MIS-C represents a potential and serious complication of COVID-19 with unspecified pathogenesis. To date, several observations suggest that MIS-C is a postinfectious, delayed immunological mediated condition or autoimmune disease that occurs after symptomatic or asymptomatic SARS-CoV-2 infection [9]. Mechanisms responsible for MIS-C, as well as the risk period, warrant future studies and should be a priority in future research.

When SARS-CoV-2 began to spread around the globe, the medical community raised concerns about the vulnerability

Grigorios Chatziparasidis

gchatziparasidis@gmail.com

1 Primary Cilia Dyskinesia Unit, School of Medicine, University of Thessaly, Thessaly, Greece

2 Pediatric Asthma and Cough Centre, Instituti Ospedalieri Bergamaschi, University and Research Hospitals, Bergamo, Italy of asthmatic children against the new virus. Presently, children with asthma do not appear to be disproportionately more affected while acquiring COVID-19. This is an evolving situation and new data may shed light on this observation. New evidence was recently summarized by Boechat et al. [10]. As previously reported, they suggested that three mechanisms may play a role: (1) the lower IFN- $\alpha$ production (ACE2 receptors upregulated by type 1 IFNs), (2) the protective role of eosinophils in the airways, and (3) the antiviral and immunomodulatory properties of ICS with their ability to downregulate ACE2 and TMPRSS2 expression. They also referred to the evidence supporting an inverse relation of allergic sensitization to ACE2 receptors. In a large-scale online survey of 91 asthma experts caring for more than 133,000 asthmatic children in five continents, $73 \%$ developed mild symptoms if infected with SARS-CoV-2, with only one child requiring hospitalization [11].

Dr. Özdemir extensively comments on the article by Moeller et al. [12]. In this paper, $70 \%$ of all asthmatic children with COVID-19 were admitted to the hospital, but only half received supplemental oxygen. Since the study took place in the early phase of the pandemic (April and May 2020), the authors found that the uncertainty about the course of COVID-19 in children may account for the high admission rate. Five asthmatic children were admitted to the PICU, in which four required ventilator support (including one teenager with acute pneumothorax and a history of mild untreated asthma). When discussing these findings, the authors state, "In general, there was a peak in PICU admissions for asthma from March to April with tree pollen season, and we speculate that some admissions may have been caused by pollen exposure and coincidental SARS-CoV-2 infection." Seasonal variation in admission to the hospital for treatment of asthma was documented in different regions of the world [13, 14], and is linked to many factors, including outdoor concentration of air pollutants and allergens [15]. In the study by Moeller et al., the authors correlate PICU admissions with pollen exposure, not the SARS-CoV-2, probably based on the previous admission data, without giving a more detailed explanation. Moeller 
et al. tried to expand on whether children with chronic respiratory illnesses have exacerbations associated with SARSCoV-2. When summarizing their findings, they mentioned that "Within participating centres for children with asthma and CF, infection with SARS-CoV- 2 was well-tolerated, but a substantial minority of children with BPD and other conditions required ventilatory support, suggesting that these groups are at risk from SARS-CoV-2 infection." These pathological conditions are beyond the scope of our paper.

Finally, we must acknowledge two errors observed by Dr Özdemir in reference 44: citing 174 centers is correct (not 147), while SARS-CoV-2 is also correct (not SARSCoV-19). We hope they do not affect the meaning of the text.

In conclusion, the risk of severe COVID-19 in subjects with asthma is influenced by multiple factors, including demographics, ethnicity, genetics, treatment, asthma severity, lung function, asthma phenotype, and comorbidities. This is an evolving research field which may uncover new mechanisms, and shed light on the long-lasting effects (longCOVID) towards improved management.

Funding None.

\section{Declarations}

Conflict of interest The authors declare no conflicts of interest.

\section{References}

1. Chatziparasidis G, Kantar A (2021) COVID-19 in Children with Asthma. Lung 199(1):7-12. https://doi.org/10.1007/ s00408-021-00419-9

2. Waterfield T, Watson C, Moore R et al (2020) Seroprevalence of SARS-CoV-2 antibodies in children-A prospective multicentre cohort study. Arch Dis Child. https://doi.org/10.1136/archdischi ld-2020-320558

3. Wu Z, McGoogan JM (2020) Characteristics of and important lessons from the coronavirus disease 2019 (COVID-19) outbreak in China: summary of a report of 72314 cases from the Chinese center for disease control and prevention. JAMA 323:1239-1242
4. Bialek S, Gierke R, Hughes $\mathrm{M}$ et al (2020) Coronavirus disease 2019 in children-United States, February 12-April 2, 2020. MMWR Morb Mortal Wkly Rep 69:422-426

5. Parri N, Lenge M, Buonsenso D (2020) Children with Covid-19 in pediatric emergency departments in Italy. $\mathrm{N}$ Engl J Med Overseas Ed 383:187-190

6. Wu C, Chen X, Cai Y et al (2020) Risk factors associated with acute respiratory distress syndrome and death in patients with coronavirus disease 2019 pneumonia in Wuhan, China. JAMA Intern Med 180(7):1-11

7. Ludvigsson JF (2020) Systematic review of COVID-19 in children shows milder cases and a better prognosis than adults. Acta Paediatr 109(6):1088-1095

8. Rubens J, Akindele N, Tschudy M, Sick-Samuels A (2021) Acute covid-19 and multisystem inflammatory syndrome in children. Brit Med J. https://doi.org/10.1136/bmj.n385

9. Nakra NA, Blumberg DA, Herrera-Guerra A, Lakshminrusimha S (2020) Multisystem inflammatory syndrome in children (MIS-C) following SARS-CoV-2 infection: Review of clinical presentation, hypothetical pathogenesis, and proposed management. Children 7:69

10. Boechat J, Wandalsen GF, Kuschnir FC, Delgado L (2021) COVID-19 and pediatric asthma: clinical and management challenges. Int J Environ Res Public Health 18:1093. https://doi.org/ 10.3390/ijerph 18031093

11. Papadopoulos N, Custovic A, Deschildre A et al (2020) Impact of COVID-19 on pediatric asthma: practice adjustments and disease burden. J Allergy Clin Immunol Pract 8:2592-2599.e3

12. Moeller A, Thanikkel L, Duijts L et al (2020) COVID-19 in children with underlying chronic respiratory diseases: survey results from 174 centres. ERJ Open Res 6:00409-02020. https://doi.org/ $10.1183 / 23120541.00409-2020$

13. Silverman R, Stevenson L, Hastings H (2003) Age-related seasonal patterns of emergency department visits for acute asthma in an urban environment. Ann Emerg Med 42(4):577-586. https:// doi.org/10.1067/S01960644(03)00410-4 (PMID: 14520329)

14. Chen C, Xirasagar S, Lin H (2006) Seasonality in adult asthma admissions, air pollutant levels, and climate: a population-based study. J Asthma 43:287-292. https://doi.org/10.1080/0277090060 0622935 (PMID: 16809242)

15. Kim K, Jahan S, Kabir E (2013) A review on human health perspectives of air pollution with respect to allergies and asthma. Environ Int 59:41-52. https://doi.org/10.1016/j.envint.2013.05. 007 (PMID: 23770580)

Publisher's Note Springer Nature remains neutral with regard to jurisdictional claims in published maps and institutional affiliations. 